\title{
The Effect of a Biomass Increase on The Carbon Fixation Rates of Arabidopsis thaliana
}

\author{
Evan Lewis ${ }^{1}$
}

${ }^{1}$ Mentor High School, Mentor, OH, USA

\section{ABSTRACT}

Arabidopsis thaliana, a model organism for genetic modification, was tested to discover whether or not a genetic increase in biomass affects the plant's rate of carbon fixation. An experimental design was employed, involving the testing of the carbon fixation rates from 4 separate experimental groups, genetically modified and a control group, both sterilized and unsterilized. Sterile water and a 50\% bleach solution were used for seed sterilization, and once complete, all types of seeds were given a week-long cold treatment. After the cold treatment had concluded, these plants were grown on moist fertilized soil, and were watered using a glass tray. Water was added into the flat surface every 2 days, and this nourishment was absorbed by the soil through holes in $4.409 \times 3.937$ terracotta pots. This absorption allowed for dry soil to remoisten, ultimately providing hydration to A. thaliana. Growth measurements of the observed largest leaf were taken each week for a 4-week growth period. Once fully grown, a procedure known as photosynthetic flotation was used to uncover carbon fixation rates between the experimental groups, involving $0.5 \mathrm{~cm}$ leaf disks rising in a bicarbonate solution as carbon dioxide was converted to oxygen. The faster the leaf disk rose, the more rapidly carbon dioxide was fixated. Overall, data gathered from this experiment showed little difference between carbon fixation rates of biomass genetic modification and a control group, causing for this genetic modification only being able to be used to combat overpopulation with larger food being produced, but not that of reversing climate change. However, there was a significant gap in terms of the time to rise of leaf disks between sterilized and unsterilized groups, revealing that a more specific study in regard to this variable must be conducted.

\section{Introduction}

"Globally, the average temperature is expected to rise another $2^{\circ} \mathrm{F}$ to $10^{\circ} \mathrm{F}$ on top of the $1^{\circ} \mathrm{F}$ increase since 1900 , according to the Intergovernmental Panel on Climate Change (IPCC)" (Meadows 2009). This immense rise in global temperature is the result of the very controversial issue of climate change. Planetary temperature increase is having a variety of unwanted outcomes, such as an intensification in the frequency of natural disasters, rise in sea level, as well as altering ecosystems due to some species' inability to adapt to a changing environment. The main cause of climate change is unequivocally the large quantities of greenhouse gases being added into the atmosphere (The IChemE Energy Centre 2015). According to NASA, these gases include nitrous oxide, water vapor, and most importantly carbon dioxide, whose main role is to hold in heat (The Effects of Climate Change 2019). With the addition of excessive amounts of their molecules into the air, a noticeable rise in temperature occurs due to miniscule quantities of heat escaping the atmosphere.

While there was an attempt to reduce greenhouse gas emissions in the form of the Paris Climate Agreement created in 2016, many large countries such as the United States chose to opt out, as they fear production loss from regulating large industries (Pulles 2017). In correspondence with climate change's effects, the grave problem of world overpopulation is only enlarging the magnitude of its consequences, due to less food in supply because of a growing society which only worsens the agricultural deficiencies resulting from changing climates. Contradictory, with a new 
genetic modification to the biomass of crops, the ramifications of these two issues can possibly be reduced in an economically and environmentally friendly way.

Genetic modification to the biomass of a plant enhances the plant's ability to grow larger. The more weight and size a plant has, the more fruits or food will be generated for consumers, providing more food to a growing society. Referencing "Overpopulation: Pakistan's Biggest Problem," sixty years ago, the population of Pakistan was 48 million with plenty of food and resources to spare, but due to overpopulation that number rose to over 190 million leading to an inability to feed thousands of people (Overpopulation 2017). This exemplification of the food inadequacy of the world portrays the necessary production increase of a variety of crops by farmers. In the same vein as this idea, Amanda Cavanuagh, a researcher who has been studying a type of biomass genetic modification that results from an alteration in the photosynthetic pathway, has increased the size of a tobacco plant by $40 \%$ (Mcgrath 2019). An enlargement of this magnitude could easily provide more food to an overpopulated society, if grown in mass quantities. However, while this research provides a way to help relieve the stress of food shortage from overpopulation, there is still no ability to aid in combating the grave dangers of climate change. In order to help draw greenhouse gas molecules from the atmosphere and possibly allow more heat to escape the planet, the increased biomass plant's carbon fixation rates must be explored.

The fixation of carbon dioxide is the process during photosynthesis in which the chloroplasts, a plant organelle, convert atmospheric carbon dioxide into oxygen, the fundamental gas required for human life (Gan et al. 2019). With more humans exhaling carbon dioxide and less photosynthesizers to fixate this gas, because of tree destruction and overpopulation, the more daunting climate change will become. While Dr. Cavanugh and her team would like to implement and share their research in the near future, USDA regulations restrict them from doing so for up to twenty years, until more research is conducted regarding the possible allergies that this specific genetic modification can bring to crops (Regalado 2019). If nothing is done in a timely manner to try to prevent the affixed complications of both food shortage caused by overpopulation and climate change consequences, these issues may be unavertable. As physicist Stephen Hawking recently remarked before his death in 2018, climate change is coming to a point where the issue will be irreversible if greenhouse gases cannot be drawn from the atmosphere, and overpopulation will eventually de-structure a starving society (Lane 2018). To possibly halt this inevitable danger and provide more food to an overpopulated world, the carbon fixation rates of a genetic modification to the biomass of plants must be tested at this current point in time to see if their mass seeding could be implemented in a more suitable time frame.

For this specific study, the plant that I will be testing is Arabidopsis thaliana, a similar species to that of a cabbage plant. Arabidopsis thalania was chosen for being an optimal subject in genetic experiments, including biomass enhancements. The main component of this plant that will be tested is the rate at which a genetic modification to the biomass of an A. thaliana, can fixate carbon dioxide into oxygen, compared to its non-genetically modified counterpart. Application of this biomass magnification was eventuated through natural accession using regional mapping collection in J. Bergelson, J. Borevitz and M. Nordborg laboratories. Natural accession is the process in which genes of one type of species, or plant variation, are applied to another. These genes can vary in number depending on what phenotype, or physical trait, is desired (Parker et al. 2016). Joy Bergelson donated her discovery to the Arabidopsis Biological Resource Center where they began producing more seeds to be shipped to the public and to me as well.

Currently, the only research that has been associated with this genetic modification is in regards to the mapping sequence used to create the growth elevation in the first place. This research was produced by The Department of Ecology and Evolution at the University of Chicago in a study titled, "Genome-Wide Patterns of Genetic Variation in Worldwide Arabidopsis thaliana Accessions from the RegMap panel," identifying the need for information in relation to carbon fixation rates of this specific biomass alteration (Horton et al. 2012). Overall, research is limited on this topic and any past studies regarding biomass genetic modifications focused on how to perfect this growth enhancement, as described in the UChicago analysis. However, now that a sufficient application has been found in the form of tobacco plants just last year under the research of Dr. Cavanugh and her colleague, Dr. Paul South, this subject is finally being brought back into the light (Temming 2019). 
Contradictory, still yet to be focused on in all of these studies regards the carbon fixation rates of this growth variation. According to a peer reviewed article titled, "Capturing Atmospheric Carbon: Biological and Nonbiological Method," carbon fixation's main goal to combat climate change is to convert atmospheric carbon dioxide during photosynthesis into a form that will not contribute to the warming of the planet (Nogia et al. 2016). If this A. thaliana genetic modification is found to have an overall increase in carbon fixation rates, then its seeding around the world in the form of edible crops could balance some of the loss of carbon sequestration over the past years due to substantial tree loss. Connectedly, the most adventitious goal of this study is to promote the use of this genetic modification in other crops, so that they too can help aid in reducing these two problems if the research succeeds. Overall, this inquiry has led to the research question, to what extent can the genetic modification to the biomass of Arabidopsis thaliana, increase the rate at which carbon dioxide is fixated?

\section{Method}

The study design that was implemented was quasi experimental, involving the testing of the photosynthetic capabilities of the largest plant material of contrasting categories. These groups consisted of: genetically modified sterile, control sterile, genetically modified non-sterile, and control non-sterile. Over a period of 4 weeks, growth rates were continuously monitored, and once plant maturation had begun to finish, the method of photosynthetic flotation was employed to gather carbon fixation rates.

In order to conduct this experiment, 2 different types of $A$. thalania were grown. These variations represented a biomass genetic modification compared to a control group. According to the ABRC, the genetically altered A. thaliana is able to grow to a height of $65 \mathrm{~cm}$ with a very large and crowded rosette, or leaf cluster, compared to the wild type, which grows on average to a height of $32 \mathrm{~cm}$ with a medium sized rosette (Arabidopsis Biological Resource Center 2020). Stock numbers purchased from the ABRC were CS78265, for the biomass genetic modification, and CS70000, for the control group. As for growing these organisms, many techniques were used to allow for the proper germination, or the beginning developmental stages of a plant and preparation of seedlings.

First, sterilization of $A$. thalania was a crucial part to the experimental process. Both the genetically modified and control group were sterilized, as well as not sterilized, for the collection of additional data in relation to plant growth rates. Seed sterilization provides for the removal of any fungal or bacterial contamination of seeds, ensuring that the plants will grow properly after germination (Rivero et al. 2013). To see instructions for how to conduct this process for A. thaliana, refer to Appendix A. Overall, around 30-40 seeds were used for sterilization of both the genetic modification and control group. Once completed, these seeds, along with the unsterilized types, were prepared for a cold treatment.

The cold treatment of A. thaliana seeds relates in a similar manner to that of seed sterilization, as both of these tasks allow for a higher seed survival rate, in addition to an increase in germination rates (Annis et al. 2014). Ultimately, this procedure is quite simple and only requires microcentrifuge tubes and sterilized water, which can be created by boiling water and allowing it to cool to room temperature. In order to carry out this ordeal, add $0.5 \mathrm{ml}$ of sterilized water to a microcentrifuge tube that contains sterilized seeds and unsterilized seeds. Next, seal the tubes that each separately contain the 4 different variable groups and place them all into the refrigerator for 1 week. After this period had expired, all seeds were ready for planting. Once the cold treatment of the 4 types of seeds was completed, the next step in the preparation for this experiment was growing and maintaining a healthy A. thaliana. Necessary tools for this preservation can be seen in Table 1 . 
Table 1. Tools Used for Growth Preparation of A. thaliana

\begin{tabular}{|l|l|}
\hline \multicolumn{1}{|c|}{ Materials } & \multicolumn{1}{c|}{ Quantity } \\
\hline Pipette & 2 \\
Sterilized Water from Boiling & 1 Pan Full \\
Plate & 1 \\
LED Growing Lights & 2 (Each Contain Two Necks, 4 Lights in Total) \\
4.409 x 3.937 Terracotta Pots & 4 (Each Contain Holes in the Bottom) \\
Flat/Tray & 1 \\
Miracle Grow Fertilized Soil & 1 Package \\
\hline Materials Found and Revised: Arabidopsis Biological Resource Center. ABRC. 2020. \\
https://abrc.osu.edu/
\end{tabular}

First, seeds were planted on the top of $4.409 \times 3.937$ terracotta pots that contained moist soil, created from mixing in small amounts of water to the soil. Approximately 15 seeds were planted per pot to allow for an innumerable amount of leaves available for the method of photosynthetic flotation. Additionally, the pots were placed on a flat, or any surface that has a square bottom but rounded edges. This tool was used to allow for the absorption of water through the holes in the terracotta plants by the soil, providing hydration to the seeds. According to Chris Calhoun, the germplasm curator of the ABRC, A. thaliana does not typically do well when watering from the top of the plant and is hence why this technique was used. The flat was covered with water daily until the seeds began to sprout. Typically, this takes around 3-7 days to occur, but once complete, water was added every two days for a period of 15 minutes. Excess water was dumped after this time and the flat was kept dry until watering was needed. In terms of a light source for these plants, 2 LED growing lamps were used that each contained two necks. These necks held a purple light lamp that could be adjusted in intensity. A consistent setting was applied for each of the pots for a 16-hour photoperiod, or an interval of time in which plants were given light. The set up for the growth of $A$. thaliana can be seen in Figure 1.

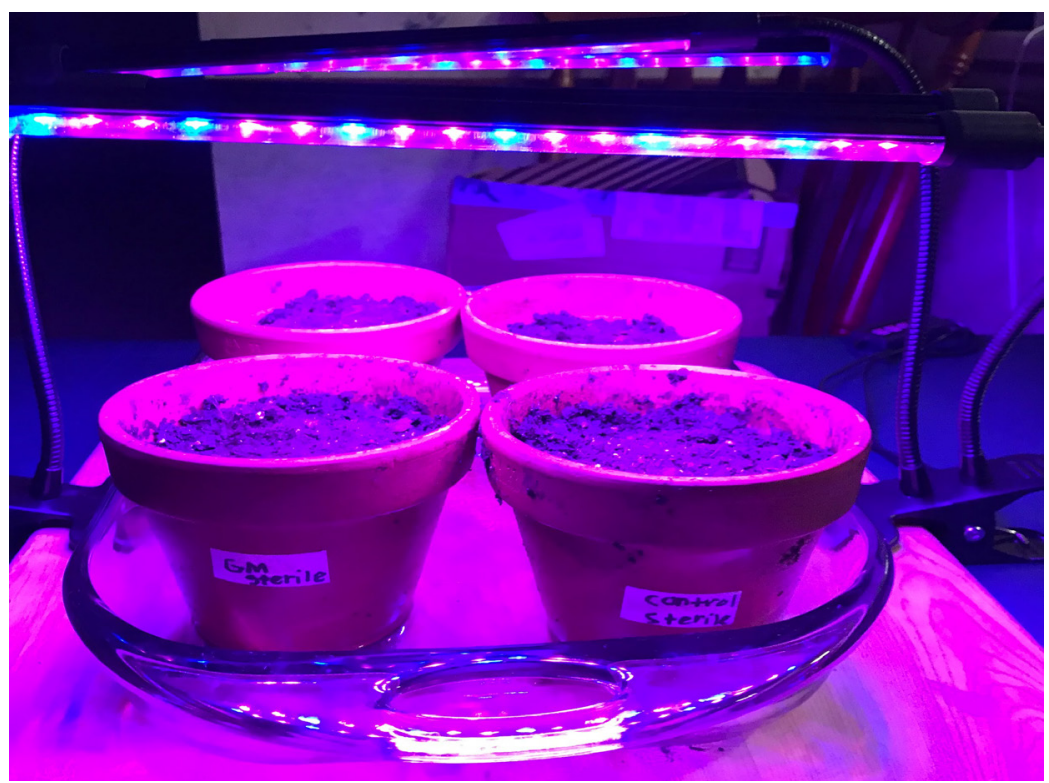

Figure 1. A. thaliana Growth Setup 
Following a period of 4 weeks of growth, in addition to the collecting of data in regards to growth development, the method of photosynthetic flotation was ready to be implemented. For this experiment, a variety of tools were needed, as displayed in Table 2.

Table 2. Tools Used for Photosynthetic Flotation

\begin{tabular}{|l|l|}
\hline \multicolumn{1}{|c|}{ Materials } & \multicolumn{1}{c|}{ Quantity } \\
\hline Baking Soda (Sodium Bicarbonate) & $\begin{array}{l}1 / 2 \text { teaspoon per 8 cups } \\
8 \text { cups } \\
\text { Water }\end{array}$ \\
Large Measuring Bowl & 1 \\
Spoon or Other Implement & 5 (In Case Straw Becomes too Wet) \\
0.5 cm Soda Straw or Hole Punch & 2 Disks Per Leaf \\
A. thaliana Leaf Disks & 1 (Preferably With a Tight Vacuum Seal) \\
10-mL Syringe (without a needle) & 4 \\
Clear Glass Cup & 1 \\
LED Light Source Used For Growth & 1 \\
Timer & \\
\hline Materials Found and Revised from: Photosynthetic Floatation. Exploratorium. 2019 Dec 4. https://www.explora- \\
torium.edu/snacks/photosynthetic-floatation
\end{tabular}

Full instructions regarding this method can be found in Appendix B. However, in short, 8 trials were conducted with 2 disks from a certain leaf being used for each of the separate experimental groups, per trial. Every disk used was made from a $0.5 \mathrm{~cm}$ straw to keep leaf size consistent among the treatment conditions. Additionally, data was collected from the time it took for the first leaf disk to float to the top of a bicarbonate solution, made by mixing water and sodium bicarbonate. These disks floated as they absorbed the carbon dioxide that was present in the solution. The faster they floated to the top of the mixture, the more rapidly carbon dioxide was fixated, allowing for carbon fixation rates to be uncovered and exemplifying how relevant this method is to the topic of inquiry. Overall, this procedure is very scholarly due to its usage around the world in biology classes, as well as in peer reviewed articles such as, "The Effect of Concentration of CO2 on the Average Rate of Photosynthesis in Spinach Leaf Disks" (Robinson-Brown 2019). However, while photosynthetic flotation may be an academic procedure due to its wide range of usage, there are some limitations associated with this method.

For example, the size of each leaf disk may not have been kept completely constant due to possible damage during the suction of air out of the surface of $A$. thaliana, as well as the fact that this experiment failed many times because of the thinness of each leaf. Additionally, this method does not exactly tell one the rate at which carbon dioxide is fixated, but instead gives a relative estimate. In spite of these limitations, most of the data that will be displayed between the treatment groups is consistent and therefore provides some reassurance that these experimental errors had little play in the collection of data. Ultimately, the key component that made this implementation successful was patience, as working with smaller, thinner plant material can be exasperating. However, through maximum preparation and trial and error, can one be successful when working with A. thalania during this experiment. 


\section{Results}

Multiple data types regarding this study can be seen in the various tables and graphs below. Table 3 displays how many seedlings sprouted out of the approximate 15 that were planted. Additionally, Figure 2 details the growth comparison of the largest leaf length for each experimental group. In terms of carbon fixation data, Table 4 displays the time to rise in seconds up the bicarbonate solution among the experimental groups, per trial. The data types are ordered from when they were collected in the experiment.

Table 3. Number of Sprouted Seeds

\begin{tabular}{|l|c|}
\hline \multicolumn{1}{|c|}{ Type of Plant } & Number of Plants Sprouted \\
\hline GM Sterile & 15 \\
\hline Control Sterile & 14 \\
\hline GM & 14 \\
\hline Control & 13 \\
\hline
\end{tabular}
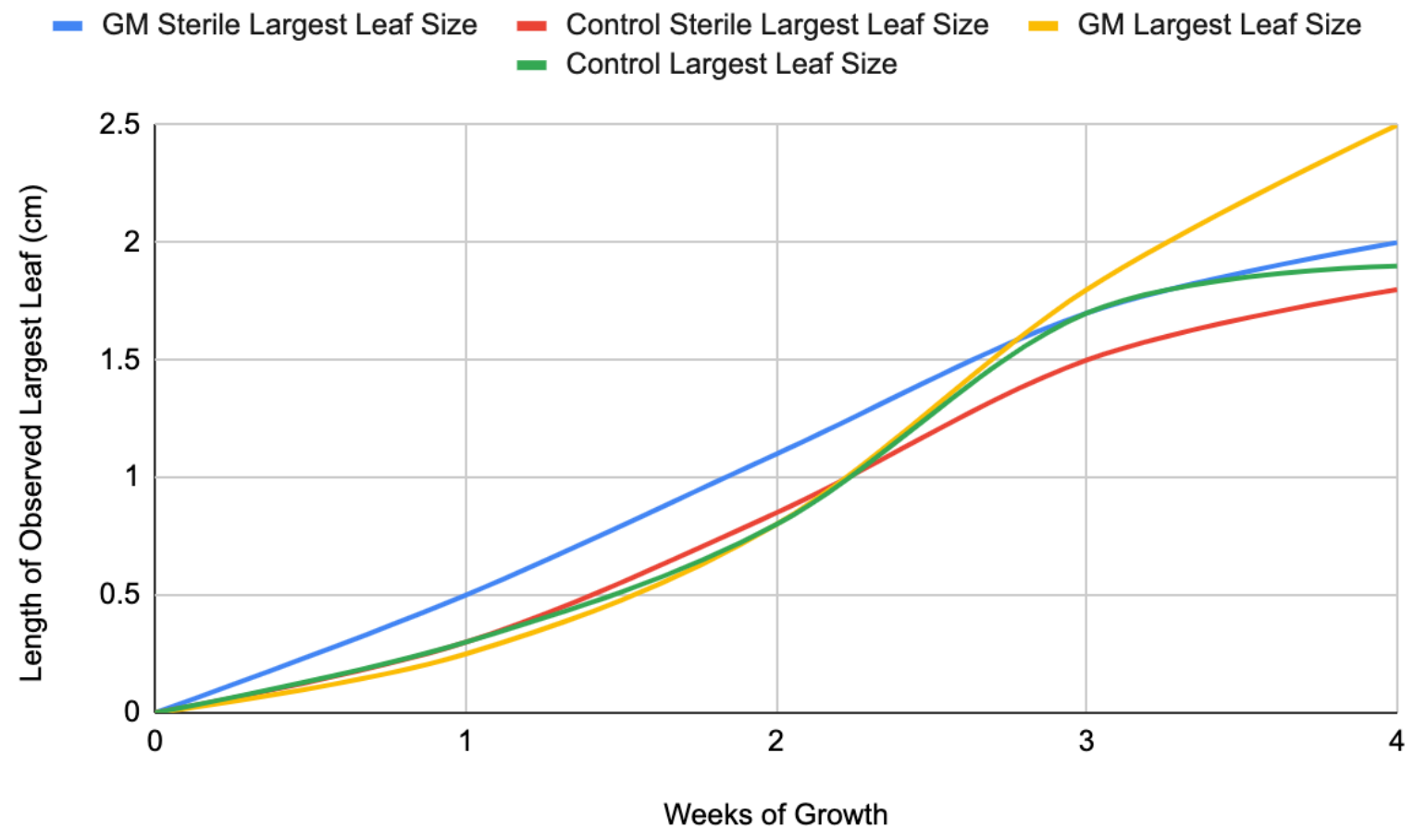

Figure 2. Growth of Different Treatment Group Leaves of A. thaliana 


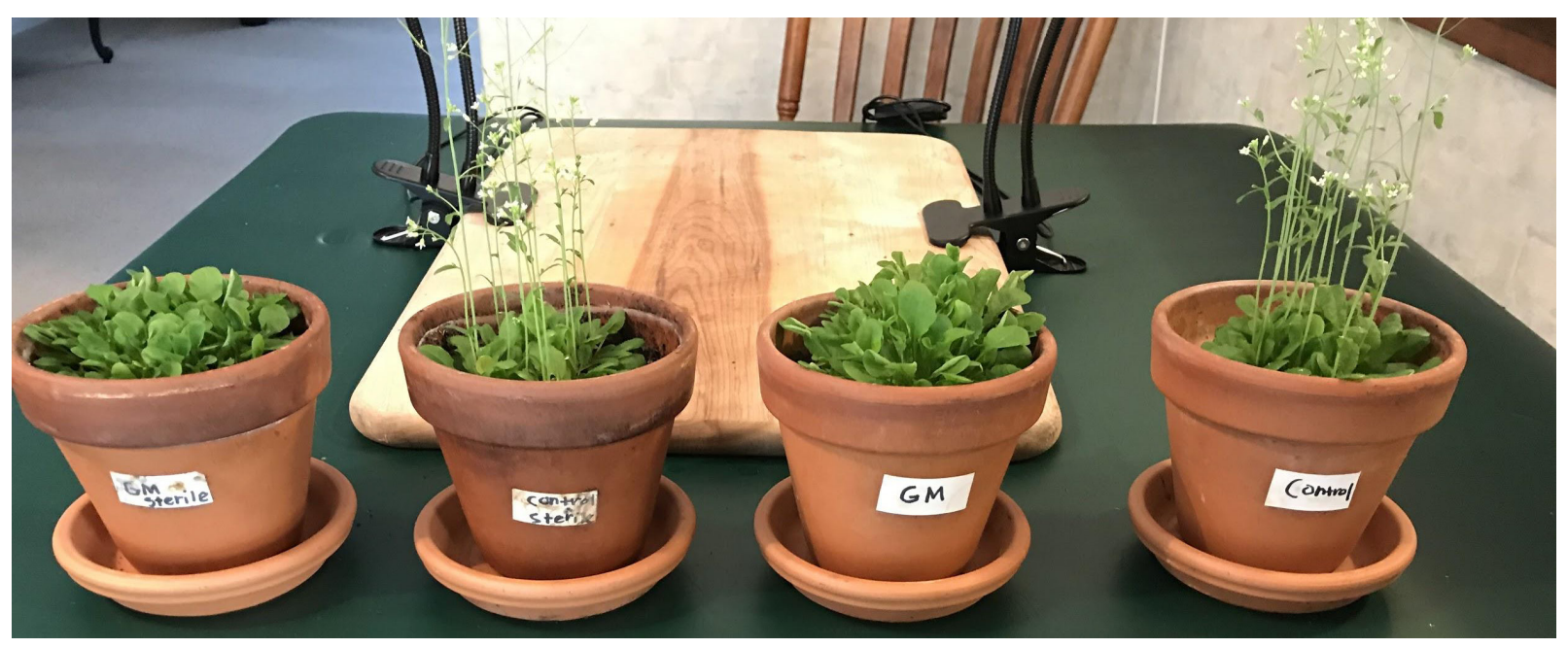

Figure 3. A. thaliana Growth Comparison

Growth rates in accordance with Figure 2 were gathered from the observed largest leaf size of each plant type as shown in Figure 3. This took place over a 4-week period and the leaves were measured every Sunday using a ruler. Figure 3 shows the final picture taken after week 4 .

Table 4. Carbon Fixation Rates Among the Experimental Groups in Seconds

\begin{tabular}{|c|c|c|c|}
\hline GM Sterile & Control Sterile & GM Unsterile & Control Unsterile \\
\hline 149.91 & 104 & 112.46 & 148.73 \\
\hline 118.93 & 191.19 & 213.56 & 168.39 \\
\hline 122.18 & 91.19 & 179.07 & 282.67 \\
\hline 216.01 & 191.82 & 207.66 & 210.91 \\
\hline 185.84 & 146.69 & 125.15 & 179.25 \\
\hline 88.3 & 135.39 & 163.43 & 161.26 \\
\hline 185.89 & 107.69 & 145.27 & 220.93 \\
\hline 111.8 & 84.16 & 141.29 & 142.16 \\
\hline & $\begin{array}{c}\text { Mean: } \\
\text { GM Sterile Mean: }\end{array}$ & GM Mean: & Control Mean: \\
\hline 147.3575 & 131.51625 & 160.98625 & 189.2875 \\
\hline
\end{tabular}


Table 5. Effect of Sterilization on Carbon Fixation Rates in Seconds

\begin{tabular}{|c|c|}
\hline Sterilized & Unsterilized \\
\hline 149.91 & 112.46 \\
\hline 118.93 & 213.56 \\
\hline 122.18 & 179.07 \\
\hline 216.01 & 207.66 \\
\hline 185.84 & 125.15 \\
\hline 88.3 & 163.43 \\
\hline 185.89 & 145.27 \\
\hline 111.8 & 141.29 \\
\hline 104 & 148.73 \\
\hline 191.19 & 168.39 \\
\hline 91.19 & 282.67 \\
\hline 191.82 & 210.91 \\
\hline 146.69 & 179.25 \\
\hline 135.39 & 161.26 \\
\hline 107.69 & 220.93 \\
\hline 84.16 & 142.16 \\
\hline Sterilized Mean: 139.436875 & Unsterilized Mean: 175.136875 \\
\hline
\end{tabular}

Table 5 displays extra data collected regarding sterilization's effect on carbon fixation rates. The two groups that were sterilized and unsterilized were added together in this graph, showing 16 total times. As one can see from the results of this table, there is a large difference in the time to rise up the bicarbonate solution among these variables. 


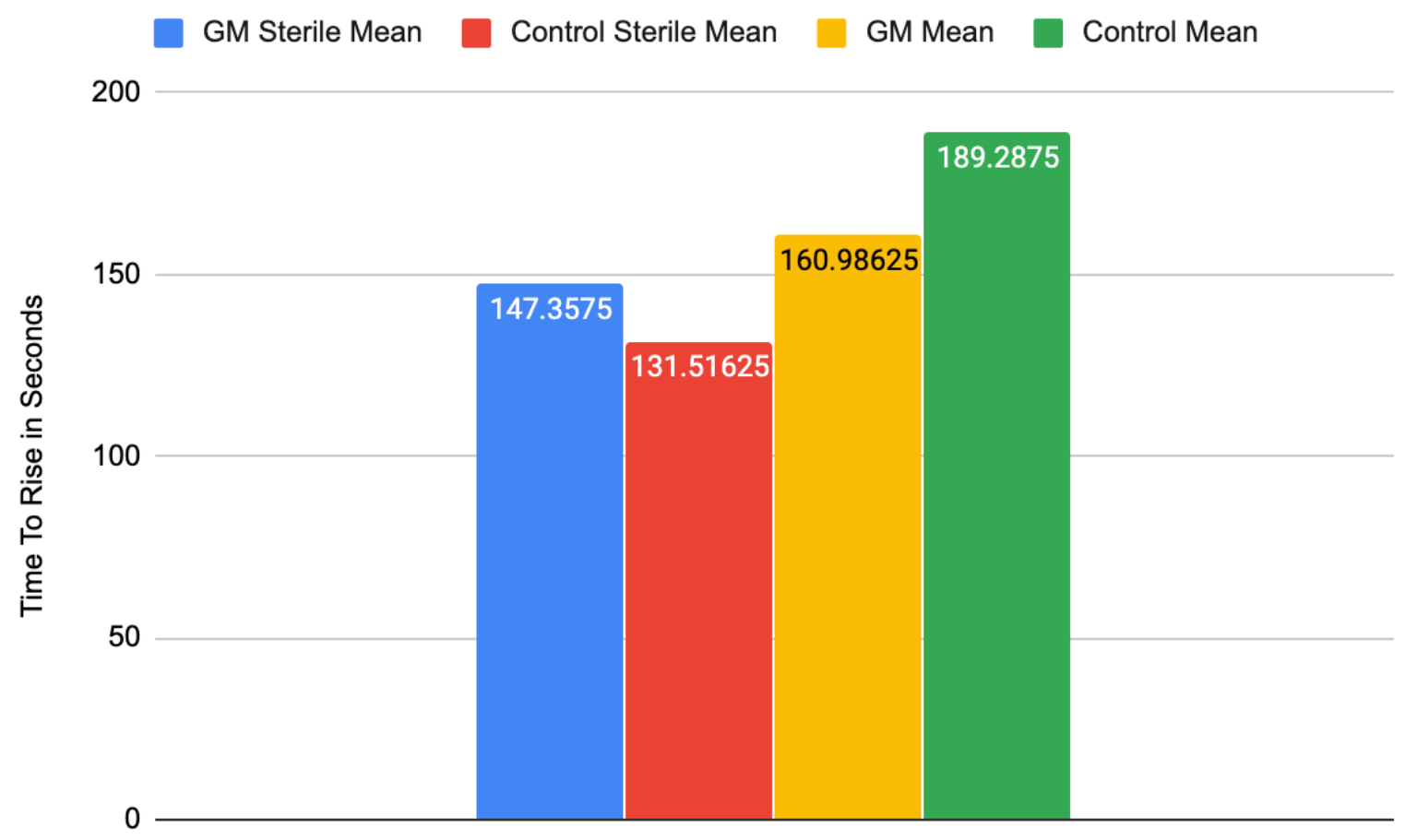

Figure 4. Average Time to Rise for Treatment Groups

Figure 4 gives a more visual graphic in relation to Table 4's data. Looking at the contrasting colors for each of the experimental groups, this bar graph gives an idea to the scale of the differences for these four variables.

\section{Analysis}

As shown by the results of the carbon fixation rates of the different variable types, there was little contrast between the time it took for a genetically modified leaf disk and a control leaf disk to rise to the top of the bicarbonate solution. This results in the conclusion being drawn that a biomass genetic modification has little to no effect on the rate at which carbon dioxide is fixated compared to the wild type. Looking at Table 4, the lowest mean time to rise for all the treatment groups was that of sterilized control with 131.51625 seconds. What this data means is that in terms of converting carbon dioxide to oxygen, a wildtype sterilized A. thaliana would be more efficient at doing so. However, in order to fully disprove the explored hypothesis, a statistical analysis must be conducted to determine the statistical significance of the data.

For this data set, a One Way ANOVA calculator was used in order to determine the p-value of the data. This type of calculation was used due to there being more than 3 separate independent variables being tested. In order for the data to be significant, the p-value needs to be less than .05 to show that there was only a $5 \%$ likelihood that the data could occur due to chance. The $\mathrm{p}$-value for the carbon fixation rate data came out to be significant at $\mathrm{p}<.1$ with a value of .070919 . Conclusively, this result leads to the conclusion that there was less than a $10 \%$ possibility that the data could have occurred from random chance and therefore is not entirely significant. Due to this outcome, the null hypothesis that states there is no statistical difference between treatment groups caused by experimental error, cannot be rejected.

Extra data that was collected during this experiment focused on the effect of sterilization on the rate at which carbon dioxide was fixated. While this data does not pertain to the main focus of this research, the findings that the 
data portrayed were very intriguing. Looking at Table 5, the time to rise for the sterilized group was 139.436875 seconds and the time to rise for the unsterilized group was 175.136875 seconds. This is almost a 40 second difference in the rate at which carbon dioxide was fixated into oxygen. When calculating the significance of this data, a T-Test calculator was used as the data type involved 2 independent measures. The results of the calculation produced a pvalue of .012934, meaning that this extra information is extremely significantly significant and was not an accidental coincidence caused by error. Additionally, this outcome gives rise to the interpretation that sterilization may have a great effect on the rate at which plants can convert $\mathrm{CO}_{2}$ into $\mathrm{O}_{2}$. Similarly, growth rates for each of the experimental groups were monitored continuously and were also collected for extra pieces of information. As one can see from Figure 2 and 3, both types of genetic modification, sterilized and unsterilized, grew larger than the control group. However, sterilization seemed to allow both the genetically modified and control group to grow larger in the first two weeks, but then were surpassed by the unsterilized groups. This data therefore provides insight that for maximum growth potential, A. thaliana needs to be unsterilized.

While this study did not produce its intended result and the data focusing on the main purpose was found to not be statistically significant, extra data regarding sterilization did produce some interesting findings. However, the limitations of this study and certain experimental errors that could have caused a statistical imbalance must be explored.

First, any leaf disks that took longer than 5 minutes to rise up the bicarbonate solution were discarded from data collection, as they were deemed to be too much of an outlier, as the average rise time was approximately 2 mins and 30 seconds. In the same vein as this idea, these outliers were thought to have occurred from an error while removing the air off of the leaf disk's surface, causing them to rise improperly. Similarly, leaf disks may not have risen rapidly because they had become stuck to the bottom of the glass cup being used and after shaking the water glass for some of these failed trials, the disks rose immediately. Supplementary tests were done to see if adequate leaf disks would rise if the water glass was moved, but the plant material did not rise, leading to the conclusion that trials over 5 minutes must have been flawed. However, I did notice that some trials did exceed this time period and rose normally after, but still the data was discarded as this was a rare occurrence. If these trials were perhaps included, then maybe the p-value of this data would have been lessened, and the status of statistically significant would have been achieved.

Another source of experimental error that could have caused the data to not be statistically significant was due to the scarcity of trials conducted. This occurred because of the difficulty of this experiment and the thinness of the plant material. 8 total trials were conducted but more would have subsequently followed if the available plant material was not scarce and there were less failed test runs. The main issue of this experiment was keeping variables constant per trial. If one experimental group had run out of functional leaves that produced two, $0.5 \mathrm{~cm}$ leaf disks, then no more trials could be run. Ultimately, this error happened after the 8th trial, leading to the end of the experiment. With more trials, could the data possibly have produced a lower p-value and less of a percent that the results produced were from chance.

Finally, the last main limitation of this study comes from the fact that there are a variety of biomass genetic modifications that have been produced and are being produced at the moment. Because this study only focuses on a specific type of genetic modification in a plant that is not in the form of a crop, this study does not speak for the broader term of a biomass increase in crops.

\section{Conclusion}

Considering all of the data and calculations, the experiment concludes that a biomass genetic modification does not have an effect on the rate at which carbon dioxide is fixed within the cells of A. thaliana. However, through a statistical significance test, the data was found to have occurred due to $7 \%$ random chance and therefore is not entirely regarded as a scientific finding. This diagnosis causes the original hypothesis, that stated that a biomass genetic modification would increase the rate at which a plant's cells could fixate carbon dioxide into oxygen, to not be discarded as more 
data will have to be collected regarding this matter. However, even with this obstacle, this study still carries some relative importance and implications for the scientific field.

To begin, my hope is that the investigation of a biomass increase will be studied more frequently around the world. Even with this experimental outcome not producing the desired results, there are still countless amounts of other types of biomass increases that can be used for testing. There could be a possibility that while this type did not have any effect on carbon fixation rates, others may have a significant effect. With this being said, I encourage other researchers to test these different types of biomass increases and their photosynthetic rates, so that there may be a possibility that this study's goal can be achieved and implemented in a more timely manner. Once other research, such as Dr. Cavanaugh's, has been completed, the problem of climate change and overpopulation will not have gotten out of hand.

Moreover, the growth rates that were gathered during A. thaliana's 4-week growth period showed that a biomass increase could grow up to $0.5 \mathrm{~cm}$ larger than the wildtype. If this increase could be applied to a much larger crop, as A. thaliana is a weed, then much larger fruit and vegetables can be produced for consumers. Of course, USDA restrictions will overall govern this implementation, but at least there could be a possibility that overpopulation can be aided in.

In closing, the last main implication of this study that carries the most importance is that of sterilizations' effect on the rate of carbon fixation and growth rates of plants as well. As shown from the results, sterilized A. thaliana rose to the top of the bicarbonate solution, on average, 40 seconds faster than the control group. This large difference indicates that sterilizing plants may in fact dramatically increase carbon fixation rates. Additionally, a T-Test showed that this data carried a p-value less than .05 , meaning that this data did not occur from chance. In a similar manner, sterilization also caused A. thaliana to grow rapidly earlier in the growth cycle and later level out, while the unsterilized groups did the exact opposite. On the contrary, my research did not focus on sterilization and therefore these claims have very little validity. However, they do give rise to a possible follow up study that only focuses on sterilization as its variable. If this study was to produce similar results, then the incitement for the creation of a healthier pesticide or sterilization source could be fabricated that would allow plants to better conduct photosynthesis and draw $\mathrm{CO}_{2}$ from the atmosphere at an increased rate. Overall, more studies and data collection will be needed to confirm the various claims throughout this investigation to possibly aid in the coming environmental detriments of the future.

\section{References}

Annis J, O'brien J, Coons J, Coutant N, Curry J. Investigation of Seed Treatments and Fertilizer on Growth of Penstemon tubaeflorus. Castanea. 2014;79(3):129-137. doi:10.2179/13-045

Arabidopsis Biological Resource Center. ABRC. 2020. https://abrc.osu.edu/

Gan P, Liu F, Li R, Wang S, Luo J. Chloroplasts- Beyond Energy Capture and Carbon Fixation: Tuning of Photosynthesis in Response to Chilling Stress. International Journal of Molecular Sciences. 2019;20(20):5046.

doi:10.3390/ijms20205046

Lane J-E. Global warming: preventing irreversibility. Brazilian Journal of Political Economy. 2018;38(4):740-748. doi:10.1590/0101-35172018-2860

Meadows R. UC scientists help California prepare for climate change. California Agriculture. 2009;63(2):56-58. doi:10.3733/ca.v063n02p56

McGrath M. Genetically modified 'shortcut' boosts plant growth by 40\%. BBC News. 2019. https://www.bbc.co.uk/news/science-environment-46750092 
Nogia, P., Sidhu, G. K., Rajesh Mehrotra, R. M., \& Mehrotra, S.. Capturing atmospheric carbon: biological and nonbiological method. International Journal of Low Carbon Technologies. 2016;11(2), 266-274. https://doi.org/10.1093/ijlct/ctt077

Overpopulation: Pakistan's biggest problem. Pulse International. 2017;18(1), 16-19. http://search.ebscohost.com/login.aspx?direct $=$ true $\& d b=a 9 h \& A N=120780426 \&$ site $=$ ehost-live

Parker N, Wang Y, Meinke D. Analysis of Arabidopsis Accessions Hypersensitive to a Loss of Chloroplast Translation. Plant Physiology. 2016;172(3):1862-1875. doi:10.1104/pp.16.01291

Photosynthetic Floatation. Exploratorium. 2019 Dec 4. https://www.exploratorium.edu/snacks/photosynthetic-floatation

Pulles T. Engineering photosynthesis: a necessary tool to protect the world's climate? Carbon Management. 2017;8(2):167-173. doi:10.1080/17583004.2017.1309201

Regalado A. Gene engineers make super-size plants that are 40\% larger. MIT Technology Review. 2019 Jan 4. https:/www.technologyreview.com/s/612710/gene-engineers-make-super-sized-plants-that-are-40-larger/

Rivero L, Scholl R, Holomuzki N, Crist D, Grotewold E, Brkljacic J. Handling Arabidopsis Plants: Growth, Preservation of Seeds, Transformation, and Genetic Crosses. Methods in Molecular Biology Arabidopsis Protocols. 013:3-25. doi:10.1007/978-1-62703-580-4_1

Robinson-Brown, A. The Effect of Concentration of CO2 on the Average Rate of Photosynthesis in Spinach Leaf Disks. Journal of the South Carolina Academy of Science, 2017;15(2), 91-93.

Temming M. A new way to genetically tweak photosynthesis boosts plant growth. Science News. 2019 Aug 8. https:/www.sciencenews.org/article/new-way-genetically-tweak-photosynthesis-boosts-plant-growth.

The Effects of Climate Change. NASA. 2019 Sep 30. https:/climate.nasa.gov/effects/

The IChemE Energy Centre Climate Communiqué. 2015. TCE: The Chemical Engineer, (892), 32-33. http://search.ebscohost.com/login.aspx?direct=true\&db=a9h\&AN=110541368\&site=ehoSt-live 